\title{
Two analytical formulae of the temperature inside a body by using partial lateral and initial data
}

\author{
Masaru IKEHATA \\ Department of Mathematics, Graduate School of Engineering \\ Gunma University, Kiryu 376-8515, JAPAN
}

October 31, 2018

\begin{abstract}
This paper considerers the problem of computing the value of a solution of the heat equation at a given point inside a bounded domain after the initial time. It is assumed that the initial value of the solution inside the domain (possibly in a part of the domain) is known; the boundary value and the normal derivative on a part of the boundary of the domain over a finite time interval are known. Two analytical formulae for the problem are given. Both formulae make use of a special fundamental solution having a large parameter of the backward heat equation.

AMS: 35R30

KEY WORDS: heat equation, backward heat equation, partial lateral and initial data, inverse heat conduction problem, ill-posed problem, enclosure method, Carleman type formula
\end{abstract}

\section{Introduction}

In this paper we consider the following inverse problem. Assume that we have a known heat conductive body and know the initial temperature inside the body (say constant). We are in the situation that we can not access the whole boundary, however, want to know the time evolution of the temperature at a given point inside the body (possibly close to an inaccessible part of the boundary) after the initial time. How can one know it from the temperature and heat flux on an accessible part of the boundary of the body for a finite observation time?

This is a typical inverse and ill-posed problem and appears in many areas of engineering and medicine. The aim of this paper is to develop an analytical approach to the problem. We consider the simplest, however, important case: the heat conductive body has a known isotropic and homogeneous heat conductivity; there is no heat source or sink inside the body. In this case, using Fourier's law, after a scaling, one may assume that the temperature at a given point and time inside the body satisfies the heat equation. Then the problem is formulated as follows.

Let $\Omega \subset \mathbf{R}^{n}(n=1,2,3)$ be a bounded domain with a smooth boundary and $0<T<$ $\infty$. Let $u=u(x, t)$ satisfy

$$
\left.\partial_{t} u=\triangle u \text { in } \Omega \times\right] 0, T[
$$


Given non empty open subsets $\Gamma \subset \partial \Omega \times] 0, T[$ and $U \subset \Omega$ (typically $U=\Omega$ ) find a formula for calculating $u(x, t)$ in $\Omega \times] 0, T[$ from the data $u(x, 0)$ for $x \in U$ and $(u(x, t), \partial u / \partial \nu(x, t))$ for $(x, t) \in \Gamma \times] 0, T[$. Here $\nu$ denotes the unit outward normal vector field to $\partial \Omega$.

We do not assume that $u(x, t)$ for $(x, t) \in(\partial \Omega \times] 0, T[) \backslash \Gamma$ and $u(x, 0)$ for $x \in \Omega \backslash U$ are known. These are considered as unknown input or output that one cannot control. In this paper we give two solutions to the problem. The consequence of the results stated in Sections 4 and 5 is the following.

Let $\left.\left(x_{0}, t_{0}\right) \in \Omega \times\right] 0, T\left[, \omega \in S^{n-1}\right.$ and $c>0$. If $(\Omega \backslash U) \times\{0\}, \Omega \times\{T\}$ and $(\partial \Omega \times] 0, T[) \backslash$ $\Gamma$ are contained in the half space $(x t)^{T} \cdot \omega(c) \leq\left(x_{0} t_{0}\right)^{T} \cdot \omega(c)-\delta$ in $\mathbf{R}^{n+1}$ with a $\delta>0$ and $\omega(c)=\left(1 / \sqrt{1+c^{2}}\right)(c \omega-1)^{T}\left(\in S^{n}\right)$, then there is an explicit formula to calculate $u\left(x_{0}, t_{0}\right)$ from the data. See Figure 1.1 for the configuration of $U, \Gamma$ and the half space $(x t)^{T} \cdot \omega(c) \leq\left(x_{0} t_{0}\right.$

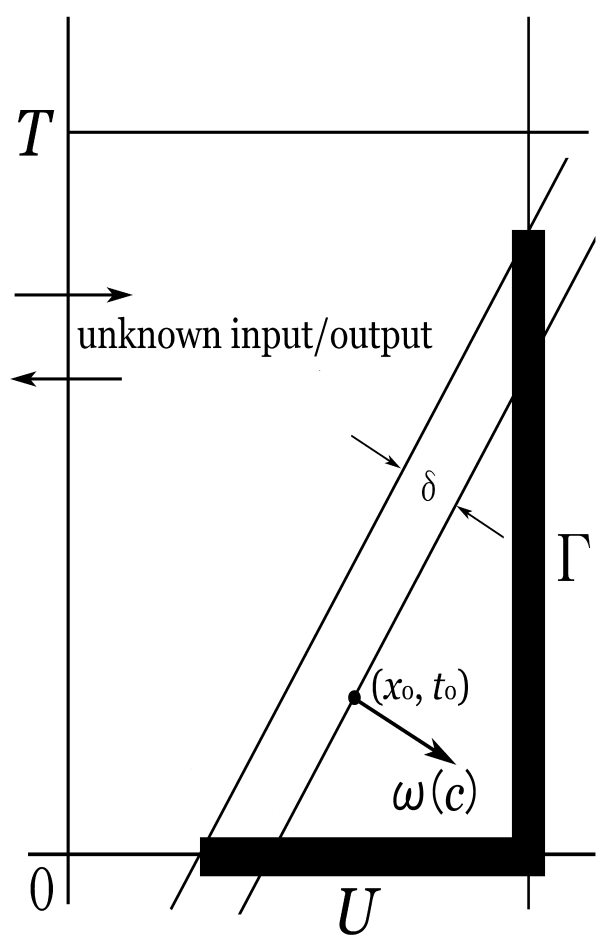

Figure 1.1: Configuration.

Here we explain our approach together with the construction of the paper.

\section{$1.1 \quad$ First approach}

First we observe that the complex exponential function $e^{x \cdot z-t(z \cdot z)}, z \in C^{n}$ satisfies the backward heat equation $\left(\partial_{t}+\triangle\right) w=0$ in $\mathbf{R}^{n+1}$. Choosing a suitable $z$ depending on a large parameter $\tau$, direction $\omega$ and $c$ (see (4.6)), we see that the real part of the phase function $x \cdot z-t(z \cdot z)$ has the form $\tau \sqrt{1+c^{2}}(x t)^{T} \cdot \omega(c)$. This yields that if $(x t)^{T} \cdot \omega(c)>0$, 
then $\left|e^{x \cdot z-t(z \cdot z)}\right| \longrightarrow \infty$ as $\tau \longrightarrow \infty$; if $(x t)^{T} \cdot \omega(c)<0$, then $\left|e^{x \cdot z-t(z \cdot z)}\right| \longrightarrow 0$ as $\tau \longrightarrow \infty$. This means that the asymptotic behaviour of $e^{x \cdot z-t(z \cdot z)}$ divides the whole space time into two parts whose common boundary is the hyper plane $(x t)^{T} \cdot \omega(c)=0$.

Second we choose $D \subset \mathbf{R}^{n+1}$ with $\left.\bar{D} \subset \Omega \times\right] 0, T\left[\right.$ and $\left(x_{0}, t_{0}\right) \in \partial D$ in such a way that for all $\rho$ smooth on $\bar{D}$ and for constants $\mu$ and $C$ independent of $\rho$

$$
\lim _{\tau \longrightarrow \infty} \tau^{\mu} e^{-x_{0} \cdot z+t_{0}(z \cdot z)} \int_{D} e^{x \cdot z-t(z \cdot z)} \rho(x, t) d x d t=C \rho\left(x_{0}, t_{0}\right) .
$$

The examples of the choice of $D$ are given in subsections in Section 4 .

Third we construct a special solution of the backward heat equation with a special inhomogeneous term:

$$
\partial_{t} v+\Delta v+e^{x \cdot z-t(z \cdot z)} \chi_{D}(x, t)=0 \text { in } \mathbf{R}^{n+1} .
$$

The point of the property of $v$ is: the growth order of a suitable norm of $e^{-x \cdot z+t(z \cdot z)} v$ as $\tau \longrightarrow \infty$ is at most algebraic. For the construction of $v$ we basically follow a Fourier transform method for a reduced equation to (1.3) which has been used in [13] since it is constructive. It starts with introducing a special fundamental solution denote by $G_{z}$ for the reduced equation. This together with necessary estimates are described in Section 3.

Finally, integrating the equation (1.1) multiplied by $v$ over $\Omega \times] 0, T$ [ and applying an integration by parts formula described in Section 2, we know that the quantity

$$
\tau^{\mu} e^{-x_{0} \cdot z+t_{0}(z \cdot z)} \int_{D} e^{x \cdot z-t(z \cdot z)} u(x, t) d x d t
$$

which is coming from the inhomogeneous term in (1.3) multiplied by $\tau^{\mu} e^{-x_{0} \cdot z+t_{0}(z \cdot z)}$, is divided into two parts: the first part consists of the data on $\Gamma$ and $U \times\{0\}$; the second unknown data on $(\Omega \backslash U) \times\{0\},(\partial \Omega \times] 0, T[) \backslash \Gamma$ and $\Omega \times\{T\}$. However, from the property of $v$ mentioned above and the assumption on the configuration of $(\Omega \backslash U) \times\{0\}$, $(\partial \Omega \times] 0, T[) \backslash \Gamma$ and $\Omega \times\{T\}$ relative to the hyper plane $(x t)^{T} \cdot \omega(c)=\left(x_{0} t_{0}\right)^{T} \cdot \omega(c)$ we know that the second part tends to 0 as $\tau \longrightarrow \infty$. Combining this with (1.2) for $\rho=u$ on $\bar{D}$, one gets a formula to calculate $u\left(x_{0}, t_{0}\right)$ from the data on $\Gamma$ and $U \times\{0\}$ only. See Section 4 for the precise description of the formula. This approach can be considered as an application of the enclosure method [8] to the heat equation (1.1).

\subsection{Second approach}

In the final section we give an integral representation formula of $G_{z}$ that is important for calculating $v$. Moreover as a byproduct we present a Carleman type formula for the heat equation, which instead of $v$, makes use of $e^{x \cdot z-t(z \cdot z)} G_{z}(x, t)$ directly which is a special fundamental solution of the backward heat equation. This approach is classical and the formula can be considered as an extension to the heat equation of Yarmukhamedov's formula [15] which has been established for the Laplace equation.

\subsection{Sideways heat equation}

There are extensive mathematical studies in the case when the one dimensional heat equation is appropriate. This case is also referred as the sideways heat equation. Carasso 
[2] considered the heat equation in the half line

$$
\left.\partial_{t} u=u_{x x} \text { in }\right] 0, \infty[\times] 0, \infty[
$$

with the initial condition $u(x, 0)=0$ for $0 \leq x<\infty$. He gave a Tikhonov regularization procedure that yields an approximation to $u(x, t)$ for $0<t<\infty$ at an arbitrary fixed $0<x<1$ from noisy $u(1, t)$ for $0 \leq t<\infty$. Note that by solving an initial boundary value problem for the heat equation in $1<x<\infty$, one gets the heat flux $u_{x}(1, t)$ from $u(1, t)$ and thus his problem is reduced to one-dimensional version of our problem. Motivated by Carasso's work Levine [11] considered a radially symmetric solution of the heat equation in higher dimensions and established a Tikhonov regularization procedure. Their methods are based on an explicit integral representation for the solution of the heat equation and not time local in the sense: to determine the value of the solution of the heat equation at a fixed point in the body one needs the data for all the time $(T=\infty)$. In contrast to their methods our method does not make use of any representation of the solution of the heat equation and needs data only on an appropriate finite time interval depending on the location of the point and time where and when we want to know the value of the solution and covers fully multi dimensional cases. For possible applications and numerical studies of the sideways heat equation, see $[1,4]$ and references therein.

\section{A weak formulation of the direct problem and in- tegration by parts formula}

In this section we describe what we mean by a solution of (1.1) together with an integration by parts formula. We follow the formulation of the direct problem described in [3].

Given $\rho \in L^{\infty}(\partial \Omega), f_{0} \in L^{2}\left(0, T ;\left(H^{1}(\Omega)\right)^{\prime}\right)$ and $h_{0} \in L^{2}\left(0, T ; H^{-1 / 2}(\partial \Omega)\right)$ we say that $u \in W\left(0, T ; H^{1}(\Omega),\left(H^{1}(\Omega)\right)^{\prime}\right)$ satisfy

$$
\begin{gathered}
\left.\partial_{t} u-\triangle u=f_{0} \text { in } \Omega \times\right] 0, T[, \\
\left.\nabla u \cdot \nu+\rho u=h_{0} \text { on } \partial \Omega \times\right] 0, T[
\end{gathered}
$$

in the weak sense if the $u$ satisfies

$$
\begin{aligned}
<u^{\prime}(t), v & >+\int_{\Omega} \nabla u(x, t) \cdot \nabla v(x) d x+\left.\left.\int_{\partial \Omega} u(t)\right|_{\partial \Omega} \cdot v\right|_{\partial \Omega} \rho d S \\
& =<f_{0}(t), v>+<h_{0}(t),\left.v\right|_{\partial \Omega}>\operatorname{in}(0, T),
\end{aligned}
$$

in the sense of distribution on $(0, T)$ for all $v \in H^{1}(\Omega)$.

Note that by Theorem 1 on p.473 in [3] we see that every $u \in W\left(0, T ; H^{1}(\Omega),\left(H^{1}(\Omega)\right)^{\prime}\right)$ is almost everywhere equal to a continuous function of $[0, T]$ in $L^{2}(\Omega)$. Further, we have:

$$
W\left(0, T ; H^{1}(\Omega),\left(H^{1}(\Omega)\right)^{\prime}\right) \hookrightarrow C^{0}\left([0, T] ; L^{2}(\Omega)\right),
$$

the space $C^{0}\left([0, T] ; L^{2}(\Omega)\right)$ being equipped with the norm of uniform convergence. Thus one can consider $u(0)$ and $u(T)$ as elements of $L^{2}(\Omega)$. Then by Theorems 1 and 2 on 
p.512 and 513 in [3] we see that given $u_{0} \in L^{2}(\Omega)$ there exists a unique $u$ such that $u$ satisfies (2.1) in the weak sense and satisfies the initial condition $u(0)=u_{0}$.

Given $\rho \in L^{\infty}(\partial \Omega), f_{1} \in L^{2}\left(0, T ;\left(H^{1}(\Omega)\right)^{\prime}\right)$ and $h_{1} \in L^{2}\left(0, T ; H^{-1 / 2}(\partial \Omega)\right)$ we say that a $v \in W\left(0, T ; H^{1}(\Omega),\left(H^{1}(\Omega)\right)^{\prime}\right)$ satisfy

$$
\begin{gathered}
\left.\partial_{t} v+\Delta v=f_{1} \text { in } \Omega \times\right] 0, T[, \\
\left.\nabla v \cdot \nu+\rho v=h_{1} \text { on } \partial \Omega \times\right] 0, T[
\end{gathered}
$$

in the weak sense if the $v$ satisfies

$$
\begin{aligned}
<v^{\prime}(t), v & >-\int_{\Omega} \nabla u(x, t) \cdot \nabla v(x) d x-\left.\left.\int_{\partial \Omega} u(t)\right|_{\partial \Omega} \cdot v\right|_{\partial \Omega} \rho d S \\
& =<f_{1}(t), v>-<h_{1}(t),\left.v\right|_{\partial \Omega}>\text { in }(0, T),
\end{aligned}
$$

in the sense of distribution on $(0, T)$ for all $v \in H^{1}(\Omega)$.

Proposition 2.1. Let $u \in W\left(0, T ; H^{1}(\Omega),\left(H^{1}(\Omega)\right)^{\prime}\right)$ satisfy (2.1) in a weak sense; let $v \in W\left(0, T ; H^{1}(\Omega),\left(H^{1}(\Omega)\right)^{\prime}\right)$ satisfy (2.3) in a weak sense. Then the formula

$$
\begin{gathered}
\int_{0}^{T}\left(<h_{1}(t),\left.u(t)\right|_{\partial \Omega}>-<h_{0}(t),\left.v(t)\right|_{\partial \Omega}>\right) d t \\
=\int_{\Omega} u(x, 0) v(x, 0) d x-\int_{\Omega} u(x, T) v(x, T) d x+\int_{0}^{T}\left(<f_{1}(t), u(t)>+<f_{0}(t), v(t)>\right) d t
\end{gathered}
$$

is valid.

Proof. Substituting $v=v(t)$ into (2.2), we have

$$
\begin{gathered}
<u^{\prime}(t), v(t)>+\int_{\Omega} \nabla u(x, t) \cdot \nabla v(x, t) d x \\
+\left.\left.\int_{\partial \Omega} u(t)\right|_{\partial \Omega} \cdot v(t)\right|_{\partial \Omega} \rho d S \\
=<f_{0}(t), v(t)>+<h_{0}(t),\left.v(t)\right|_{\partial \Omega}>\text { a.e.in }(0, T) .
\end{gathered}
$$

Similarly we have also

$$
\begin{gathered}
<v^{\prime}(t), u(t)>-\int_{\partial \Omega} \nabla v(x, t) \cdot \nabla u(x, t) d x \\
-\left.\left.\int_{\partial \Omega} v(t)\right|_{\partial \Omega} \cdot u(t)\right|_{\partial \Omega} \rho d S \\
=<f_{1}(t), u(t)>-<h_{1}(t),\left.u(t)\right|_{\partial \Omega}>\text { a.e. } \operatorname{in}(0, T) .
\end{gathered}
$$

Taking the sum of (2.5) and (2.6), we have

$$
\begin{aligned}
<u^{\prime}(t), v(t)> & +<v^{\prime}(t), u(t)>=<f_{0}(t), v(t)>+<h_{0}(t),\left.v(t)\right|_{\partial \Omega}> \\
& +<f_{1}(t), u(t)>-<h_{1}(t),\left.u(t)\right|_{\partial \Omega}>.
\end{aligned}
$$


Integrating both sides of this equation over the interval $(0, T)$ and using the formula (Theorem 2 on p.477 in [3])

$$
\begin{aligned}
& \int_{0}^{T}<u^{\prime}(t), v(t)>d t+\int_{0}^{T}<v^{\prime}(t), u(t)>d t \\
& =\int_{\Omega} u(x, T) v(x, T) d x-\int_{\Omega} u(x, 0) v(x, 0) d x
\end{aligned}
$$

we obtain (2.4).

In particular, let $u \in W\left(0, T ; H^{1}(\Omega),\left(H^{1}(\Omega)\right)^{\prime}\right)$ satisfy

$$
\begin{gathered}
\left.\partial_{t} u-\triangle u=0 \text { in } \Omega \times\right] 0, T[, \\
\left.\nabla u \cdot \nu+\rho u=h_{0} \text { on } \partial \Omega \times\right] 0, T[
\end{gathered}
$$

in the weak sense and $v \in W\left(0, T ; H^{1}(\Omega),\left(H^{1}(\Omega)\right)^{\prime}\right)$ satisfy

$$
\begin{gathered}
\left.\partial_{t} v+\triangle v=f_{1} \text { in } \Omega \times\right] 0, T[, \\
\left.\nabla v \cdot \nu+\rho v=h_{1} \text { on } \partial \Omega \times\right] 0, T[
\end{gathered}
$$

in the weak sense. Then (2.4) gives the formula

$$
\begin{gathered}
\int_{0}^{T}\left(<h_{1}(t),\left.u(t)\right|_{\partial \Omega}>-<h_{0}(t),\left.v(t)\right|_{\partial \Omega}>\right) d t \\
=\int_{0}^{T}<f_{1}(t), u(t)>d t+\int_{\Omega} u(x, 0) v(x, 0) d x-\int_{\Omega} u(x, T) v(x, T) d x .
\end{gathered}
$$

\section{A solution of the equation $\partial_{t} v+\Delta v+f=0$}

In this section given $f=f(x, t)$ we construct a special solution of the equation

$$
-\partial_{t} v-\triangle v=f \text { in } \mathbf{R}^{n+1}
$$

Note that $-\partial_{t}-\triangle$ is the formal adjoint for $\partial_{t}-\triangle$.

Given a complex vector $z \in \boldsymbol{C}^{n}$ set

$$
v(x, t)=e^{x \cdot z-t(z \cdot z)} w(x, t), \quad f(x, t)=e^{x \cdot z-t(z \cdot z)} g(x, t) .
$$

Then (3.1) becomes

$$
-\partial_{t} w-2 z \cdot \nabla w-\triangle w=g .
$$

Taking the Fourier transform of both sides, we obtain

$$
\left(-i \eta-2 i z \cdot \xi+|\xi|^{2}\right) \hat{w}(\xi, \eta)=\hat{g}(\xi, \eta) .
$$

This motivates us to study the meaning of $1 / P_{z}(\xi, \eta)$ where

$$
P_{z}(\xi, \eta)=-i \eta-2 i z \cdot \xi+|\xi|^{2}
$$


Let

$$
z=\boldsymbol{a}+i \boldsymbol{b}, \boldsymbol{a}, \boldsymbol{b} \in \mathbf{R}^{n} .
$$

Since

$$
P_{z}(\xi, \eta)=|\xi+\boldsymbol{b}|^{2}-|\boldsymbol{b}|^{2}-i(\eta+2 \boldsymbol{a} \cdot \xi),
$$

we have: $P_{z}(\xi, \eta)=0$ if and only if $|\xi+\boldsymbol{b}|=|\boldsymbol{b}|$ and $\eta+2 \boldsymbol{a} \cdot \xi=0$. Therefore the set $\left\{(\xi, \eta) \in \mathbf{R}^{n+1} \mid P_{z}(\xi, \eta)=0\right\}$ is a compact set and forms a submanifold of $\mathbf{R}^{n+1}$ with codimension 2 provided $\boldsymbol{b} \neq 0$. This yields that for $z \in \boldsymbol{C}^{n}$ with $\operatorname{Im} z \neq 0$ the function $1 / P_{z}(\xi, \eta)$ defines a tempered distribution on $\mathbf{R}^{n+1}$.

Definition 3.1. Define $G_{z}(x, t)$ as the inverse Fourier transform of $1 / P_{z}(\xi, \eta)$ :

$$
G_{z}(x, t)=\frac{1}{(2 \pi)^{n+1}} \int e^{i(x \cdot \xi)+i t \eta} \frac{d \xi d \eta}{P_{z}(\xi, \eta)}, \operatorname{Im} z \neq 0 .
$$

In this section we study the convolution operator $g \mapsto G_{z} * g$ acting on the set of all rapidly decreasing functions on $\mathbf{R}^{n+1}$.

First we restrict ourself to the case when $\operatorname{Re} z=0$. More precisely, given $\omega \in S^{n-1}$ and $\tau>0$ set

$$
z=i \tau \omega .
$$

We set $F_{\tau}(x, t)=G_{z}(x, t)$ for this $z$, that is

$$
F_{\tau}(x, t)=\frac{1}{(2 \pi)^{n+1}} \int e^{i(x \cdot \xi)+i t \eta} \frac{d \xi d \eta}{-i \eta+2 \tau \omega \cdot \xi+|\xi|^{2}} .
$$

Let us study the property of $F_{\tau}(x, t)$. For the purpose we employ the argument done in [7]. The points are: a relationship between the operators $F_{\tau} * \cdot$ and $F_{1} * \cdot$; an estimation of a scaling effect on weighted $L^{2}$-norms; an weighted $L^{2}$-estimate for the operator $F_{1} *$.

\subsection{Scaling laws}

It is easy to see that $F_{\tau}(x, t)$ satisfies the scaling law:

$$
\forall \lambda>0 F_{\lambda \tau}(x, t)=\lambda^{n} F_{\tau}\left(\lambda x, \lambda^{2} t\right) .
$$

Given a distribution $g(x, t)$ define

$$
g_{\lambda}(x, t)=g\left(\lambda x, \lambda^{2} t\right), \lambda>0 .
$$

It is easy to see that $\left(g_{\lambda^{-1}}\right)_{\lambda}=g$. From (3.4) we have

$$
\begin{aligned}
& F_{\tau} * g(x, t)=\int F_{\tau}(x-y, t-s) g(y, s) d y d s \\
& =\tau^{n} \int F_{1}\left(\tau(x-y), \tau^{2}(t-s)\right) g(y, s) d y d s .
\end{aligned}
$$

This yields

$$
\begin{gathered}
\left(F_{\tau} * g\right)\left(\tau^{-1} x, \tau^{-2} t\right)=\tau^{n} \int F_{1}\left(x-\tau y, t-\tau^{2} s\right) g(y, s) d y d s \\
=\frac{1}{\tau^{2}} \int F_{1}(x-y, t-s) g\left(\tau^{-1} y, \tau^{-2} s\right) d y d s
\end{gathered}
$$


That is

$$
\left(F_{\tau} * g\right)_{\tau^{-1}}=\frac{1}{\tau^{2}} F_{1} *\left(g_{\tau^{-1}}\right)
$$

or equivalently

$$
F_{\tau} * g=\frac{1}{\tau^{2}}\left\{F_{1} *\left(g_{\tau^{-1}}\right)\right\}_{\tau}
$$

This yields also

$$
D_{x}^{\alpha} D_{t}^{\beta} F_{\tau} * g=\tau^{-2+|\alpha|+2 \beta}\left\{D_{x}^{\alpha} D_{t}^{\beta} F_{1} *\left(g_{\tau^{-1}}\right)\right\}_{\tau} .
$$

Given $s \in \mathbf{R}$ we denote by $L_{s}^{2}\left(\mathbf{R}^{n+1}\right)$ the set of all tempered distributions $g=g(x, t)$ that satisfies $\left(1+|x|^{2}+t^{2}\right)^{s / 2} g \in L^{2}\left(\mathbf{R}^{n+1}\right)$ and set

$$
\|g\|_{s}=\left(\int|g(x, t)|^{2}\left(1+|x|^{2}+t^{2}\right)^{s} d x d t\right)^{1 / 2}, s \in \mathbf{R} .
$$

Note that the set of all rapidly decreasing functions on $\mathbf{R}^{n+1}$ is dense in $L_{s}^{2}\left(\mathbf{R}^{n+1}\right)$.

Given $R>0$ let $\tau \geq R$. Set

$$
C(R)=\min \left\{R^{4}, R^{2}, 1\right\}(>0) .
$$

Then we have

$$
\begin{gathered}
1+\frac{|x|^{2}}{\tau^{2}}+\frac{t^{2}}{\tau^{4}}=\frac{1}{\tau^{4}}\left(\tau^{4}+\tau^{2}|x|^{2}+t^{2}\right) \\
\geq \frac{1}{\tau^{4}}\left(R^{4}+R^{2}|x|^{2}+t^{2}\right) \\
\geq \frac{C(R)}{\tau^{4}}\left(1+|x|^{2}+t^{2}\right)
\end{gathered}
$$

Since

$$
\begin{aligned}
& \left\|g_{\tau}\right\|_{s}^{2}=\int\left|g\left(\tau x, \tau^{2} t\right)\right|^{2}\left(1+|x|^{2}+t^{2}\right)^{s} d x d t \\
& =\frac{1}{\tau^{n+2}} \int|g(x, t)|^{2}\left(1+\frac{|x|^{2}}{\tau^{2}}+\frac{t^{2}}{\tau^{4}}\right)^{s} d x d t
\end{aligned}
$$

from (3.7) one gets

$$
\left\|g_{\tau}\right\|_{s} \leq \frac{C(R)^{s / 2}}{\tau^{2 s+(n+2) / 2}}\|g\|_{s}, s<0
$$

For $\tau \geq R$ we have

$$
\begin{aligned}
1+\tau^{2}|x|^{2} & +\tau^{4} t^{2}=\tau^{4}\left(\tau^{-4}+\tau^{-2}|x|^{2}+t^{2}\right) \\
& \leq \tau^{4}\left(\frac{1}{R^{4}}+\frac{|x|^{2}}{R^{2}}+t^{2}\right) \\
& \leq \frac{\tau^{4}}{C(R)}\left(1+|x|^{2}+t^{2}\right)
\end{aligned}
$$

Let $s^{\prime}>0$. Since

$$
\left\|g_{\tau^{-1}}\right\|_{s^{\prime}}^{2}=\tau^{n+2} \int|g(x, t)|^{2}\left(1+\tau^{2}|x|^{2}+\tau^{4} t^{2}\right)^{s^{\prime}} d x d t
$$


from (3.9) we obtain

$$
\left\|g_{\tau^{-1}}\right\|_{s^{\prime}} \leq \frac{\tau^{2 s^{\prime}+(n+2) / 2}}{C(R)^{s^{\prime} / 2}}\|g\|_{s^{\prime}}
$$

\subsection{Weighted $L^{2}$-estimates}

Lemma 3.1. Let $-1<\delta<0$. Given a rapidly decreasing function $g$ on $\mathbf{R}^{n+1}$ the tempered distribution $F_{1} * g$ belongs to $L_{\delta}^{2}\left(\mathbf{R}^{n+1}\right)$ and there exists a positive constant $C_{\delta}$ independent of $g$ and $\omega$ such that

$$
\left\|D_{x}^{\alpha} D_{t}^{\beta} F_{1} * g\right\|_{\delta} \leq C_{\delta}\|g\|_{1+\delta},|\alpha|+2 \beta \leq 2 .
$$

Proof. For $z=i \omega$, we have $P_{z}(\xi, \eta)=|\xi+\omega|^{2}-1-i \eta$. Let $|\xi| \geq 8$. We have

$$
\begin{gathered}
\left|P_{z}(\xi, \eta)\right|^{2}=\left(|\xi|^{2}+2 \xi \cdot \omega\right)^{2}+\eta^{2} \\
=|\xi|^{4}+4(\xi \cdot \omega)|\xi|^{2}+4(\xi \cdot \omega)^{2}+\eta^{2} \\
\geq|\xi|^{4}+\eta^{2}-4|\xi|^{3}=|\xi|^{4}\left(1-\frac{4}{|\xi|}\right)+\eta^{2} \\
\geq \frac{1}{2}|\xi|^{4}+\eta^{2} \geq \frac{1}{2}\left(|\xi|^{4}+\eta^{2}\right) \geq \frac{1}{4}\left(|\xi|^{2}+|\eta|\right)^{2} .
\end{gathered}
$$

Next let $|\xi| \leq 8$ and $|(\xi, \eta)| \geq 8 \sqrt{1+8^{2}}$. We have

$$
|\eta|^{2} \geq 8^{2}\left(1+8^{2}\right)-|\xi|^{2} \geq 8^{4} \geq|\xi|^{4} .
$$

This yields $|\eta| \geq\left(|\xi|^{2}+|\eta|\right) / 2$ and thus one gets

$$
\left|P_{z}(\xi, \eta)\right|^{2} \geq \eta^{2} \geq\left(\frac{1}{2}\right)^{2}\left(|\xi|^{2}+|\eta|\right)^{2} .
$$

Therefore it holds that, for all $(\xi, \eta) \in \mathbf{R}^{n+1}$ with $|(\xi, \eta)| \geq 8 \sqrt{1+8^{2}}$

$$
\left|P_{z}(\xi, \eta)\right| \geq \frac{1}{2}\left(|\xi|^{2}+|\eta|\right) \text {. }
$$

Using this inequality, a local representation of $1 / P_{z}(\xi, \eta)$ in each neighbourhood of some zero points of $P_{z}(\xi, \eta)$ and Lemma 3.1 in [13], we have the desired conclusion.

A combination of (3.5), (3.6), (3.8), (3.10) and (3.11) yields

Proposition 3.1. Let $-1<\delta<0$ and $R>0$. For all rapidly decreasing functions $g$ on $\mathbf{R}^{n+1}$ and $\tau \geq R$ we have

$$
\left\|D_{x}^{\alpha} D_{t}^{\beta} G_{i \tau \omega} * g\right\|_{\delta} \leq \frac{C_{\delta} \tau^{|\alpha|+2 \beta}}{C(R)^{1 / 2}}\|g\|_{1+\delta},|\alpha|+2 \beta \leq 2 .
$$


Proof. First consider the case when $|\alpha|=\beta=0$. We have

$$
\begin{gathered}
\left\|F_{\tau} * g\right\|_{\delta}=\frac{1}{\tau^{2}}\left\|\left\{F_{1} *\left(g_{\tau^{-1}}\right)\right\}_{\tau}\right\|_{\delta} \\
\leq \frac{C(R)^{\delta / 2}}{\tau^{2 \delta+2+(n+2) / 2}}\left\|G_{1} *\left(g_{\tau^{-1}}\right)\right\|_{\delta} \\
\leq \frac{C(R)^{\delta / 2} C_{\delta}}{\tau^{2 \delta+2+(n+2) / 2}}\left\|g_{\tau^{-1}}\right\|_{1+\delta} \\
\leq \frac{C(R)^{\delta / 2} C_{\delta}}{\tau^{2 \delta+2+(n+2) / 2}} \frac{\tau^{2(1+\delta)+(n+2) / 2}}{C(R)^{(1+\delta) / 2}}\|g\|_{1+\delta}
\end{gathered}
$$

Since we have (3.6), a similar argument yields (3.12) for the case when $|\alpha| \neq 0$ or $\beta \neq 0$.

For general $z$ the following property is the starting point.

Proposition 3.2. For all $z \in \boldsymbol{C}^{n}$ with $\operatorname{Im} z \neq 0$ we have

$$
G_{z}(x, t)=G_{i m z}(x-2 t \operatorname{Re} z, t) .
$$

Proof. Let $z=\boldsymbol{a}+i \boldsymbol{b}$. From (3.3) we have

$$
G_{z}(x, t)=\frac{1}{(2 \pi)^{n+1}} \int e^{i(x \cdot \xi)+i t \eta} \frac{d \xi d \eta}{-i(\eta+2 \boldsymbol{a} \cdot \xi)+2 \boldsymbol{b} \cdot \xi+|\xi|^{2}} .
$$

Then change of variables

$$
\eta^{\prime}=\eta+2 \boldsymbol{a} \cdot \xi, \xi^{\prime}=\xi
$$

yields

$$
G_{z}(x, t)=\frac{1}{(2 \pi)^{n+1}} \int e^{i\left(x \cdot \xi^{\prime}\right)+i t\left(\eta^{\prime}-2 \boldsymbol{a} \cdot \xi^{\prime}\right)} \frac{d \xi^{\prime} d \eta^{\prime}}{-i \eta^{\prime}-2 i(i \boldsymbol{b}) \cdot \xi^{\prime}+\left|\xi^{\prime}\right|^{2}}
$$

Since

$$
i\left(x \cdot \xi^{\prime}\right)+i t\left(\eta^{\prime}-2 \boldsymbol{a} \cdot \xi^{\prime}\right)=i(x-2 t \boldsymbol{a}) \cdot \xi^{\prime}+i t \eta^{\prime},
$$

we obtain the desired formula.

Now given a real vector $\boldsymbol{c}$ set

$$
g_{\boldsymbol{c}}(x, t)=g(x-t \boldsymbol{c}, t)
$$

From (3.13) we see that, for $z=\boldsymbol{a}+i \boldsymbol{b}$

$$
\begin{aligned}
& \left(G_{z} * g\right)(x, t)=\int G_{z}(x-y, t-s) g(y, s) d y d s \\
& =\int G_{i \boldsymbol{b}}(x-y-2(t-s) \boldsymbol{a}, t-s) g(y, s) d y d s .
\end{aligned}
$$


This yields that

$$
\begin{gathered}
\left(G_{z} * g\right)(x+2 t \boldsymbol{a}, t)=\int G_{i \boldsymbol{b}}(x-y+2 s \boldsymbol{a}, t-s) g(y, s) d y d s \\
=\int G_{i} \boldsymbol{b}\left(x-y^{\prime}, t-s\right) g\left(y^{\prime}+2 s \boldsymbol{a}, s\right) d y^{\prime} d s
\end{gathered}
$$

and thus we have

$$
\left(G_{z} * g\right)_{-2} \boldsymbol{a}=G_{i \boldsymbol{b}} *\left(g_{-2} \boldsymbol{a}\right) .
$$

Since $\left(g_{\boldsymbol{c}}\right)_{-\boldsymbol{c}}=g$, from $(3.14)$ we obtain

$$
G_{z} * g=\left\{G_{i \boldsymbol{b}} *\left(g_{-2} \boldsymbol{a}\right)\right\}_{2} \boldsymbol{a}
$$

and also

$$
\begin{gathered}
D_{x}^{\alpha}\left(G_{z} * g\right)=\left(D_{x}^{\alpha} G_{i} \boldsymbol{b}^{* 2} \boldsymbol{a}\right)_{2} \boldsymbol{a} \\
D_{t}\left(G_{z} * g\right)=-\sum_{j=1}^{n}\left(\frac{\partial}{\partial x_{j}} G_{i} \boldsymbol{b}^{* 2} g_{-2}\right)_{2} 2 a_{j}+\left(D_{t} G_{i} \boldsymbol{b}^{*} g_{-2} \boldsymbol{a}\right)_{2} \boldsymbol{a}
\end{gathered}
$$

Remark 3.1. The equation (3.15) corresponds to the simple fact: a function $w(x, t)$ satisfies the equation (3.2) if and only if the function $\tilde{w}(x, t) \equiv w(x+2 t \boldsymbol{a}, t)$ satisfies the equation

$$
-\partial_{t} \tilde{w}-2 i \boldsymbol{b} \cdot \nabla \tilde{w}-\triangle \tilde{w}=g(x+2 t \boldsymbol{a}, t) .
$$

Now we give an estimate for $G_{z} * g$.

Theorem 3.1. Let $-1<\delta<0$ and $R>0$. Let $z=\boldsymbol{a}+i \boldsymbol{b}$. For all rapidly decresing functions $g$ on $\mathbf{R}^{n+1}$ and $\boldsymbol{b} \neq 0$ with $|\boldsymbol{b}| \geq R$ we have

$$
\begin{gathered}
\left\|D_{x}^{\alpha} G_{z} * g\right\|_{\delta} \leq C(R, \delta)\left(\sqrt{1+|\boldsymbol{a}|^{2}}+|\boldsymbol{a}|\right)|\boldsymbol{b}|^{|\alpha|}\|g\|_{\delta+1},|\alpha| \leq 2 \\
\left\|D_{t} G_{z} * g\right\|_{\delta} \leq C(R, \delta)\left(\sqrt{1+|\boldsymbol{a}|^{2}}+|\boldsymbol{a}|\right)\left(2|\boldsymbol{a}||\boldsymbol{b}|+|\boldsymbol{b}|^{2}\right)\|g\|_{\delta+1}
\end{gathered}
$$

Proof. It suffices to consider only the case when $\boldsymbol{a} \neq 0$. Let $|\alpha|=0$. Set $f=G_{i \boldsymbol{b}^{*}}\left(g_{-2 \boldsymbol{a}}\right)$. From (3.15) we have

$$
\begin{gathered}
\left\|G_{z} * g\right\|_{\delta}^{2}=\left\|f_{2 \boldsymbol{a}}\right\|_{\delta}^{2} \\
=\int|f(x-2 t \boldsymbol{a}, t)|^{2}\left(1+|x|^{2}+t^{2}\right)^{\delta} d x d t \\
=\int|f(y, t)|^{2}\left(1+|y+2 t \boldsymbol{a}|^{2}+t^{2}\right)^{\delta} d y d t .
\end{gathered}
$$

One can write

$$
|y+2 t \boldsymbol{a}|^{2}+t^{2}=A\left(\begin{array}{l}
y \\
t
\end{array}\right) \cdot\left(\begin{array}{l}
y \\
t
\end{array}\right)
$$


where

$$
A=\left(\begin{array}{cc}
I_{n} & 2 \boldsymbol{a} \\
2 \boldsymbol{a}^{T} & 1+4|\boldsymbol{a}|^{2}
\end{array}\right) \text {. }
$$

It is easy to see that the eigenvalues $\lambda$ of $A$ coincides with the roots of the equation

$$
\lambda^{2}-2\left(1+2|\boldsymbol{a}|^{2}\right) \lambda+1=0 .
$$

Solving this equation, we have

$$
\lambda=\left(1+2|\boldsymbol{a}|^{2}\right) \pm 2|\boldsymbol{a}| \sqrt{1+|\boldsymbol{a}|^{2}} .
$$

Since the minimum eigenvalue has the form

$$
\left(1+2|\boldsymbol{a}|^{2}\right)-2|\boldsymbol{a}| \sqrt{1+|\boldsymbol{a}|^{2}}=\frac{1}{\left(\sqrt{1+|\boldsymbol{a}|^{2}}+|\boldsymbol{a}|\right)^{2}},
$$

one has

$$
\frac{|y|^{2}+t^{2}}{\left(\sqrt{1+|\boldsymbol{a}|^{2}}+|\boldsymbol{a}|\right)^{2}} \leq A\left(\begin{array}{c}
y \\
t
\end{array}\right) \cdot\left(\begin{array}{c}
y \\
t
\end{array}\right) \leq\left(\sqrt{1+|\boldsymbol{a}|^{2}}+|\boldsymbol{a}|\right)^{2}\left(|y|^{2}+t^{2}\right) .
$$

Since $-1<\delta<0$, from (3.17) we have

$$
\left\|f_{2 \boldsymbol{a}}\right\|_{\delta}^{2} \leq\left(\sqrt{1+|\boldsymbol{a}|^{2}}+|\boldsymbol{a}|\right)^{-2 \delta}\|f\|_{\delta}^{2}
$$

and

$$
\left\|g_{-2 \boldsymbol{a}}\right\|_{\delta+1}^{2} \leq\left(\sqrt{1+|\boldsymbol{a}|^{2}}+|\boldsymbol{a}|\right)^{2(1+\delta)}\|g\|_{\delta+1}^{2} .
$$

These together with (3.12) yield

$$
\begin{gathered}
\\
\left\|G_{z} * g\right\|_{\delta}^{2} \leq\left(\sqrt{1+|\boldsymbol{a}|^{2}}+|\boldsymbol{a}|\right)^{-2 \delta}\|f\|_{\delta}^{2} \\
\leq C(R, \delta)\left(\sqrt{1+|\boldsymbol{a}|^{2}}+|\boldsymbol{a}|\right)^{-2 \delta}\|g-2 \boldsymbol{a}\|_{\delta+1}^{2} \\
\leq C(R, \delta)\left(\sqrt{1+|\boldsymbol{a}|^{2}}+|\boldsymbol{a}|\right)^{-2 \delta+2(1+\delta)}\|g\|_{\delta+1}^{2} .
\end{gathered}
$$

Other cases also can be proved as above since we have (3.16).

Therefore the map $g \mapsto G_{z} * g \in L_{\delta}^{2}\left(\mathbf{R}^{n+1}\right)$ can be uniquely extended as a bounded linear operator of $L_{\delta+1}^{2}\left(\mathbf{R}^{n+1}\right)$ into $L_{\delta}^{2}\left(\mathbf{R}^{n+1}\right)$. We denote it by the same symbol. Then we see that, given $z$ with $\operatorname{Im} z \neq 0$ and $g \in L_{\delta+1}^{2}\left(\mathbf{R}^{n+1}\right)$ the the function

$$
v(x, t)=e^{x \cdot z-t(z \cdot z)}\left(G_{z} * g\right)(x, t)
$$


satisfies the backward heat equation with a source term in the sense of distribution:

$$
\partial_{t} v+\triangle v+e^{x \cdot z-t(z \cdot z)} g=0 \text { in } \mathbf{R}^{n+1} .
$$

Note that $e^{-x \cdot z+t(z \cdot z)} v(x, t) \in L_{\delta}^{2}\left(\mathbf{R}^{n+1}\right)$ and this $v$ is unique. This is a consequence of Theorem 7.1.27 of [6] and the facts that the set of all zero points of $P(\xi, \eta)$ has codimension 2 in $\mathbf{R}^{n+1}$ and $-1<\delta<0$. See also Corollary 3.4 in [13] for this type of argument.

Remark 3.2. Hsieh[5] developed the scattering theory associated with the operator $\partial_{t}-\triangle$ in $\mathbf{R}^{2+1}$. For the purpose he studied the operator $L^{1}\left(\mathbf{R}^{2+1}\right) \ni f \mapsto\left(1 / Q_{z}(\xi, \eta)\right) \hat{q} * f \in$ $L^{1}\left(\mathbf{R}^{2+1}\right)$ where $\hat{q}$ the Fourier transform of a function $q$ on $\mathbf{R}^{2+1}, z \in \boldsymbol{C}^{2}$ and $Q_{z}(\xi, \eta)$ the symbol of the operator $e^{-x \cdot z-t(z \cdot z)}\left(\partial_{t}-\triangle\right) e^{x \cdot z+t(z \cdot z)}$. Note that $e^{x \cdot z+t(z \cdot z)}$ satisfies the heat equation not the backward heat equation. In the paper there is no result related with Theorem 3.1.

\section{A computation formula of $u$ in $R^{n+1}$ with $n=1,2,3$}

In this section we assume that $h_{0}$ in $(2.7)$ satisfies $h_{0} \in L^{2}\left(0, T ; L^{2}(\partial \Omega)\right)$ not just $h_{0} \in$ $L^{2}\left(0, T ; H^{-1 / 2}(\partial \Omega)\right)$.

Let $u \in W\left(0, T ; H^{1}(\Omega),\left(H^{1}(\Omega)\right)^{\prime}\right)$ satisfy $(2.7)$ in the weak sense. Let $D$ a bounded open subset of $\mathbf{R}^{n+1}$ with $\left.\bar{D} \subset \Omega \times\right] 0, T\left[\right.$. We denote by $\chi_{D}$ the characteristic function of $D$. Let $v$ be the distribution given by (3.18) with $g=\chi_{D}$.

Now choose a sequence $g_{j} \in C_{0}^{\infty}\left(\mathbf{R}^{n+1}\right)$ in such a way that $g_{j} \longrightarrow \chi_{D}$ in $L_{\delta+1}^{2}\left(\mathbf{R}^{n+1}\right)$ as $j \longrightarrow \infty$. Define

$$
v_{j}(x, t)=e^{x \cdot z-t(z \cdot z)}\left(G_{z} * g_{j}\right)(x, t) .
$$

A combination of Theorem 3.1 and the Sobolev imbedding theorem in $\mathbf{R}^{n+1}$ we see that $v_{j} \in C^{\infty}\left(\mathbf{R}^{n+1}\right)$ and $\left\{\left.v_{j}\right|_{\Omega \times] 0, T[}\right\}$ is Cauchy in $H^{2,1}(\Omega \times] 0, T[) \equiv L^{2}\left(0, T ; H^{2}(\Omega)\right) \cap$ $H^{1}\left(0, T ; L^{2}(\Omega)\right)$ (see pages $6-7$ in [12]). Since $\left.\left.v_{j}\right|_{\Omega \times] 0, T[} \longrightarrow v\right|_{\Omega \times] 0, T[}$ in $L^{2}(\Omega \times] 0, T[)$, we conclude that $v \in H^{2,1}(\Omega \times] 0, T[)$ and this $v$ satisfies

$$
\left\|D_{x}^{\alpha} D_{t}^{\beta}\left(e^{-x \cdot z+t(z \cdot z)} v(x, t)\right)\right\|_{L^{2}(\Omega \times] 0, T[)}=O\left(|z|^{3}\right),|\alpha|+2 \beta \leq 2 .
$$

By the trace theorem (Theorem 2.1 on p. 9 in [12]) we have $\left.\left.v_{j}\right|_{\partial \Omega \times] 0, T[} \longrightarrow v\right|_{\partial \Omega \times] 0, T[}$ in $H^{3 / 2,3 / 4}(\partial \Omega \times] 0, T[) \equiv L^{2}\left(0, T ; H^{3 / 2}(\partial \Omega)\right) \cap H^{3 / 4}\left(0, T ; L^{2}(\partial \Omega)\right) ; \partial v_{j} /\left.\partial \nu\right|_{\partial \Omega \times] 0, T[} \longrightarrow$ $\partial v /\left.\partial \nu\right|_{\partial \Omega \times] 0, T[}$ in $H^{1 / 2,1 / 4}(\partial \Omega \times] 0, T[) \equiv L^{2}\left(0, T ; H^{1 / 2}(\partial \Omega)\right) \cap H^{1 / 4}\left(0, T ; L^{2}(\partial \Omega)\right)$; $v_{j}(x, 0) \longrightarrow v(x, 0)$ and $v_{j}(x, T) \longrightarrow v(x, T)$ in $H^{1}(\Omega)$.

Note that $v_{j} \in W\left(0, T ; H^{1}(\Omega),\left(H^{1}(\Omega)\right)^{\prime}\right)$ and satisfies (2.8) in the weak sense with $f_{1}=$ $-e^{x \cdot z-t(z \cdot z)} g_{j}(x, t)$ and $h_{1}=\partial v_{j} / \partial \nu+\rho v_{j}$ on $\left.\partial \Omega \times\right] 0, T[$.

Thus (2.9) yields

$$
\begin{gathered}
\int_{0}^{T} \int_{\partial \Omega}\left\{\left.\left(\frac{\partial v_{j}}{\partial \nu}+\rho v_{j}\right) u(t)\right|_{\partial \Omega}-\left.h_{0}(t) v_{j}(t)\right|_{\partial \Omega}\right\} d S d t \\
=-\int_{0}^{T} \int_{\Omega} e^{x \cdot z-t(z \cdot z)} g_{j}(x) u(x, t) d x d t+\int_{\Omega} u(x, 0) v_{j}(x, 0) d x-\int_{\Omega} u(x, T) v_{j}(x, T) d x .
\end{gathered}
$$


Taking the limit $j \longrightarrow \infty$, we obtain

$$
\begin{gathered}
\int_{\partial \Omega \times] 0, T[}\left\{\left(\frac{\partial v}{\partial \nu}(x, t)+\rho(x) v(x, t)\right) u(x, t)-h_{0}(x, t) v(x, t)\right\} d S d t \\
+\int_{\Omega} u(x, T) v(x, T) d x-\int_{\Omega} u(x, 0) v(x, 0) d x \\
=-\int_{D} e^{x \cdot z-(z \cdot z) t} u(x, t) d x d t
\end{gathered}
$$

Note that we made use of the fact that: every $\phi \in L^{2}\left(0, T ; L^{2}(\partial \Omega)\right)$ can be identified with $\phi(x, t) \equiv \phi(t)(x) \in L^{2}(\partial \Omega \times] 0, T[)$.

Divide $\partial \Omega \times] 0, T[=\Gamma \cup\{(\partial \Omega \times] 0, T[) \backslash \Gamma\}$ and $\Omega=U \cup(\Omega \backslash U)$. Define

$$
\begin{aligned}
I(\tau)=\int_{\Gamma}\left\{\left(\frac{\partial v}{\partial \nu}(x, t)\right.\right. & \left.+\rho(x) v(x, t)) u(x, t)-h_{0}(x, t) v(x, t)\right\} d S d t \\
& -\int_{U} v(x, 0) u(x, 0) d x .
\end{aligned}
$$

From (4.3) we have

$$
I(\tau)=-\int_{D} e^{x \cdot z-(z \cdot z) t} u(x, t) d x d t+R
$$

where

$$
\begin{gathered}
R=-\int_{\Omega} v(x, T) u(x, T) d x+\int_{\Omega \backslash U} v(x, 0) u(x, 0) d x \\
-\int_{\partial \Omega \times] 0, T \backslash \backslash \Gamma}\left\{\left(\frac{\partial v}{\partial \nu}(x, t)+\rho(x) v(x, t)\right) u(x, t)-h_{0}(x, t) v(x, t)\right\} d S d t .
\end{gathered}
$$

Given $c>0$ and $\omega \in S^{n-1}$ define

$$
\omega(c)=\frac{1}{\sqrt{1+c^{2}}}\left(\begin{array}{c}
c \omega \\
-1
\end{array}\right) \in S^{n}
$$

and for $\tau$ with $c^{2} \tau>1$ set

$$
z= \begin{cases}c \tau\left(\omega+i \sqrt{1-\frac{1}{c^{2} \tau}} \omega^{\perp}\right), \omega^{\perp} \in S^{n-1} & \text { if } n=2,3 \\ c \tau\left(1+i \sqrt{1-\frac{1}{c^{2} \tau}}\right) \omega, \omega=\{1,-1\} & \text { if } n=1 .\end{cases}
$$

One can write

$$
\operatorname{Re}\{x \cdot z-t(z \cdot z)\}=\tau \sqrt{1+c^{2}}\left(\begin{array}{l}
x \\
t
\end{array}\right) \cdot \omega(c)
$$


since $\operatorname{Re} z \cdot z=\tau$.

In our method the concept introduced in the following plays an important role.

Definition 4.1. We say that $D$ is visible at $\left(x_{0}, t_{0}\right) \in \mathbf{R}^{n+1}$ as $\tau \longrightarrow \infty$ from the complex direction $z$ given by (4.6) if there exist $\mu>0$ and constant $C \neq 0$ such that for all $\rho \in C^{\infty}(\bar{D})$

$$
\lim _{\tau \longrightarrow \infty} e^{-x_{0} \cdot z+t_{0}(z \cdot z)} \tau^{\mu} \int_{D} e^{x \cdot z-t(z \cdot z)} \rho(x, t) d x d t=C \rho\left(x_{0}, t_{0}\right) .
$$

The constant $C$ is unique if it exists.

Theorem 4.1. Let $\left.\left(x_{0}, t_{0}\right) \in \Omega \times\right] 0, T[$ be an arbitrary fixed point. Assume that $T>0$, $\omega, \Gamma$ and $U$ satisfy the following conditions:

$$
\begin{aligned}
& \sup _{x \in \Omega}\left(\begin{array}{c}
x \\
T
\end{array}\right) \cdot \omega(c)<\left(\begin{array}{c}
x_{0} \\
t_{0}
\end{array}\right) \cdot \omega(c) ; \\
& \sup _{x \in \Omega \backslash U}\left(\begin{array}{c}
x \\
0
\end{array}\right) \cdot \omega(c)<\left(\begin{array}{c}
x_{0} \\
t_{0}
\end{array}\right) \cdot \omega(c) ; \\
& \sup _{(x, t) \in(\partial \Omega \times] 0, T[\backslash \backslash \Gamma}\left(\begin{array}{c}
x \\
t
\end{array}\right) \cdot \omega(c)<\left(\begin{array}{c}
x_{0} \\
t_{0}
\end{array}\right) \cdot \omega(c) .
\end{aligned}
$$

Assume that $D$ with $\bar{D} \subset \Omega \times] 0, T\left[\right.$ is visible at $\left(x_{0}, t_{0}\right)$ from the complex direction $z$ given by (4.6). Let $v$ be given by (3.18) with $g=\chi_{D}$. Then we have

$$
u\left(x_{0}, t_{0}\right)=-\frac{1}{C} \lim _{\tau \longrightarrow \infty} \tau^{\mu} e^{-x_{0} \cdot z+t_{0}(z \cdot z)} I(\tau),
$$

where

$$
I(\tau)=\int_{\Gamma}\left\{\left(\frac{\partial v}{\partial \nu}(x, t)+\rho(x) v(x, t)\right) u(x, t)-h_{0}(x, t) v(x, t)\right\} d S d t-\int_{U} v(x, 0) u(x, 0) d x .
$$

Proof. Assumptions (4.8), (4.9), (4.10) together with the trace theorem, (4.2) and (4.5) ensure that $\left|\tau^{\mu} e^{-x_{0} \cdot z+t_{0}(z \cdot z)} R\right|$ is decaying as $\tau \longrightarrow \infty$. Since the heat operator $\partial_{t}-\triangle$ is hypoelliptic, we know $u \in C^{\infty}(\bar{D})$. This together with (4.4) and (4.7) yields (4.11).

The formula (4.11) can be considered as an application of an idea in [8,9] that was originally developed for the Cauchy problem for the stationary Schrödinger equation.

So the problem is reduced to: how to choose $D$ that is visible at $\left(x_{0}, t_{0}\right)$ from the complex direction $z$. In the following subsections we consider this problem.

\subsection{The case $n=1$}

Let $\delta>0$. We denote by $D\left(x_{0}, t_{0}, \omega(c), \delta\right)$ the inside of the triangle with vertices $P=$ $\left(x_{0}, t_{0}\right), P_{0}=\left(x_{0}-(\delta / c) \sqrt{1+c^{2}} \omega, t_{0}\right)$ and $P_{1}=\left(x_{0}, t_{0}+\delta \sqrt{1+c^{2}}\right)$ in the space time $\mathbf{R}^{1+1}$. The two points $P_{1}$ and $P_{2}$ are located on the line $(x, t)^{T} \cdot \omega(c)=\left(x_{0}, t_{0}\right)^{T} \cdot \omega(c)-\delta$. 
In [10] we have already known the following. For the proof see the proof of theorem 2.3 of in [10].

Proposition 4.1. Let $D=D\left(x_{0}, t_{0}, \omega(c), \delta\right)$. If $\rho \in C^{2}(\bar{D})$, then

$$
\lim _{\tau \longrightarrow \infty} 2(c \tau)^{2} \tau e^{-x_{0} \cdot z+t_{0}(z \cdot z)} \int_{D} e^{x \cdot z-t(z \cdot z)} \rho(x, t) d x d t=-\frac{i\left|P_{1}-P_{0}\right|^{2} \rho(P)}{\left|P_{1}-P\right|\left(\sqrt{c^{2}+1}+i(c / \delta)\left|P_{0}-P\right|\right)} .
$$

Therefore this $D$ is visible at $\left(x_{0}, t_{0}\right)$ from complex direction $z$. The constants $\mu$ and $C$ in (4.7) are given by $\mu=3$ and

$$
C=-\frac{1+i}{4 c^{3}}
$$

\subsection{The cases when $n=2,3$}

The cases when $n=2,3$ start with describing the following which is easily derived by the proof of Theorem 2.2 and Lemma 4.1 in [10].

Proposition 4.2. Let $n \geq 2$. Let $D \subset \mathbf{R}^{n+1}$ be a finite cone with a vertex at $P=\left(x_{0}, t_{0}\right)$ and a bottom face $Q \neq \emptyset$ that is a bounded open subset of $n$-dimensional hyper plane $(x t)^{T} \cdot \omega(c)=\left(x_{0} t_{0}\right)^{T} \cdot \omega(c)-\delta$. If $\rho \in C^{0, \theta}(\bar{D})$ with $0<\theta \leq 1$, then

$$
\lim _{\tau \longrightarrow \infty} \frac{2}{n !}(c \tau)^{n+1} e^{-x_{0} \cdot z+t(z \cdot z)} \int_{D} e^{x \cdot z-t(z \cdot z)} \rho(x, t) d x d t=K_{D} \rho(P)
$$

where

$$
K_{D}=2 \delta \int_{Q} \frac{d S(y)}{\left(\frac{\delta \sqrt{c^{2}+1}}{c}-i(y-P) \cdot\left(\begin{array}{c}
\omega^{\perp} \\
0
\end{array}\right)\right)^{n+1}}
$$

Therefore if $K_{D} \neq 0$, then constants $\mu$ and $C$ in (4.7) are given by $\mu=n+1$ and

$$
C=\frac{n ! K_{D}}{2 c^{n+1}}
$$

However, it is not easy to show that $K_{D} \neq 0$ for $D$ with general $Q$. In the following we specify $Q$ and show that $K_{D} \neq 0$.

\subsubsection{The case when $n=2$}

Let $\delta>0$. Choose arbitrary two points $x_{1}, x_{2}$ on the line $x \cdot \omega=x_{0} \cdot \omega-(\delta / c) \sqrt{1+c^{2}}$ in such a way that the orientation of the two vectors $\omega, x_{1}-x_{2}$ coincides with that of the standard basis $\boldsymbol{e}_{1}, \boldsymbol{e}_{2}$. We denote by $D\left(x_{0}, x_{1}, x_{2}, \omega(c), \delta\right)$ the inside of the tetrahedron in $\mathbf{R}^{2+1}$ with the vertices $\left(x_{0}, t_{0}\right),\left(x_{1}, t_{0}\right),\left(x_{2}, t_{0}\right)$ and $\left(x_{0}, t_{0}+\delta \sqrt{1+c^{2}}\right)$. We see that the three points $\left(x_{1}, t_{0}\right),\left(x_{2}, t_{0}\right)$ and $\left(x_{0}, t_{0}+\delta \sqrt{1+c^{2}}\right)$ are on the plane $(x t)^{T} \cdot \omega(c)=\left(x_{0}, t_{0}\right) \cdot \omega(c)-\delta$. Therefore $D\left(x_{0}, x_{1}, x_{2}, \omega(c), \delta\right)$ coincides with the finite cone with a vertex at $\left(x_{0}, t_{0}\right)$ and a bottom face $Q$ that is the triangle in $\mathbf{R}^{2+1}$ with the vertices $\left(x_{1}, t_{0}\right),\left(x_{2}, t_{0}\right)$ and $\left(x_{0}, t_{0}+\delta \sqrt{1+c^{2}}\right)$. 
From (4.2) in [10] we obtain the formula

$$
\begin{gathered}
K_{D}=K_{D} \vartheta \cdot(0-1)^{T} \\
=c^{3} \sum_{j=1}^{3} \frac{\left|\left(\boldsymbol{\nu}_{j} \times \boldsymbol{\nu}_{j-1}\right) \times\left(\boldsymbol{\nu}_{j+1} \times \boldsymbol{\nu}_{j}\right)\right|}{\left.\left.\left\{\left(\boldsymbol{\nu}_{j} \times \boldsymbol{\nu}_{j-1}\right) \cdot \vartheta\right)\right\}\left\{\left(\boldsymbol{\nu}_{j+1} \times \boldsymbol{\nu}_{j}\right) \cdot \vartheta\right)\right\}} \boldsymbol{\nu}_{j} \cdot(0-1)^{T}
\end{gathered}
$$

where $D=D\left(x_{0}, x_{1}, x_{2}, \omega(c), \delta\right), \boldsymbol{\nu}_{1}=\boldsymbol{\nu}_{4}, \boldsymbol{\nu}_{2}, \boldsymbol{\nu}_{3}=\boldsymbol{\nu}_{0}$ are the unit outward normal vector to the faces of $D$ that are triangles $\Delta_{1}$ with the vertices $\left(x_{0}, t_{0}\right),\left(x_{1}, t_{0}\right),\left(x_{0}, t_{0}+\right.$ $\left.\delta \sqrt{1+c^{2}}\right), \Delta_{2}$ with the vertices $\left(x_{0}, t_{0}\right),\left(x_{2}, t_{0}\right),\left(x_{0}, t_{0}+\delta \sqrt{1+c^{2}}\right), \Delta_{3}$ with the vertices $\left(x_{0}, t_{0}\right),\left(x_{1}, t_{0}\right),\left(x_{2}, t_{0}\right) ; \vartheta=\left(c\left(\omega+i \omega^{\perp}\right)-1\right)^{T}$.

Since Corollary 4.1 in [10] ensures this $K_{D} \neq 0$, we conclude that $D$ is visible at $\left(x_{0}, t_{0}\right)$ from the complex direction $z$. Note that $\boldsymbol{\nu}_{3}=(0-1)^{T}$ and $\boldsymbol{\nu}_{1} \cdot \boldsymbol{\nu}_{3}=\boldsymbol{\nu}_{2} \cdot \boldsymbol{\nu}_{3}=0$. Therefore from (4.13) and (4.14) we have the simpler expression

$$
C=\frac{\left|\left(\boldsymbol{\nu}_{3} \times \boldsymbol{\nu}_{2}\right) \times\left(\boldsymbol{\nu}_{1} \times \boldsymbol{\nu}_{3}\right)\right|}{\left.\left.\left\{\left(\boldsymbol{\nu}_{3} \times \boldsymbol{\nu}_{2}\right) \cdot \vartheta\right)\right\}\left\{\left(\boldsymbol{\nu}_{1} \times \boldsymbol{\nu}_{3}\right) \cdot \vartheta\right)\right\}} .
$$

Now choose $x_{1}, x_{2}, \delta$ in such a way that $\left.\overline{D\left(x_{0}, x_{1}, x_{2}, \omega(c), \delta\right)} \subset \Omega \times\right] 0, T[$. Then we obtain the formula (4.11) for $D=D\left(x_{0}, x_{1}, x_{2}, \omega(c), \delta\right), \mu=3$ and $C$ given by (4.15).

\subsubsection{The case when $n=3$}

Let $\delta>0$. Choose arbitrary three points $x_{1}, x_{2}$ and $x_{3}$ on the plane $x \cdot \omega=x_{0} \cdot \omega-$ $(\delta / c) \sqrt{1+c^{2}}$ in such a way that the orientation of the three vectors $\omega, x_{1}-x_{2}, x_{3}-x_{2}$ coincides with that of the standard basis $\boldsymbol{e}_{1}, \boldsymbol{e}_{2}, \boldsymbol{e}_{3}$. The four points $x_{0}, x_{1}, x_{2}$ and $x_{3}$ form a tetrahedron $\Delta$ in $\mathbf{R}^{3}$. We denote by $\boldsymbol{\nu}$ the unit outward normal vector field to $\partial \Delta$. $\partial \Delta$ consists of four triangles: $T_{1}$ with the vertices $x_{0}, x_{1}$ and $x_{2} ; T_{2}$ with the vertices $x_{0}$, $x_{3}$ and $x_{2} ; T_{3}$ with the vertices $x_{0}, x_{1}$ and $x_{3} ; T_{4}$ with the vertices $x_{1}, x_{2}$ and $x_{3}$. Since $\boldsymbol{\nu}$ takes a constant vector on each $T_{j}$, we denote the vector by $\boldsymbol{\nu}_{j}$. In particular, we have $\boldsymbol{\nu}_{4}=-\omega$.

We denote by $D\left(x_{0}, x_{1}, x_{2}, x_{3}, \omega(c), \delta\right)$ the inside of the finite cone in $\mathbf{R}^{3+1}$ with a vertex $\left(x_{0}, t_{0}+\delta \sqrt{1+c^{2}}\right)$ and the bottom that is the inside of the tetrahedron in the space $t=t_{0}$ with vertices $\left(x_{0}, t_{0}\right),\left(x_{1}, t_{0}\right),\left(x_{2}, t_{0}\right)$ and $\left(x_{3}, t_{0}\right)$. Then the boundary of $D\left(x_{0}, x_{1}, x_{2}, x_{3}, \omega(c), \delta\right)$ consists of five tetrahedrons: $\Delta_{1}$ with the vertices $\left(x_{0}, t_{0}\right),\left(x_{1}, t_{0}\right)$, $\left(x_{2}, t_{0}\right)$ and $\left(x_{0}, t_{0}+\delta \sqrt{1+c^{2}}\right) ; \Delta_{2}$ with the vertices $\left(x_{0}, t_{0}\right),\left(x_{2}, t_{0}\right),\left(x_{3}, t_{0}\right)$ and $\left(x_{0}, t_{0}+\right.$ $\left.\delta \sqrt{1+c^{2}}\right) ; \Delta_{3}$ with the vertices $\left(x_{0}, t_{0}\right),\left(x_{3}, t_{0}\right),\left(x_{1}, t_{0}\right)$ and $\left(x_{0}, t_{0}+\delta \sqrt{1+c^{2}}\right) ; \Delta_{4}$ with the vertices $\left(x_{0}, t_{0}\right),\left(x_{1}, t_{0}\right),\left(x_{2}, t_{0}\right)$ and $\left(x_{3}, t_{0}\right) ; Q$ with vertices $\left(x_{1}, t_{0}\right),\left(x_{2}, t_{0}\right),\left(x_{3}, t_{0}\right)$ and $\left(x_{0}, t_{0}+\delta \sqrt{1+c^{2}}\right)$.

We see that $D=D\left(x_{0}, x_{1}, x_{2}, x_{3}, \omega(c), \delta\right)$ coincides with the finite cone with a vertex at $\left(x_{0}, t_{0}\right)$ and the bottom $Q$. Let $\boldsymbol{a}$ be an arbitrary constant vector in $\boldsymbol{C}^{3+1}$. Since

$$
\nabla_{(x, t)} \cdot\left(e^{x \cdot z-t(z \cdot z)} \boldsymbol{a}\right)=(z-\tau)^{T} \cdot \boldsymbol{a} e^{x \cdot z-t(z \cdot z)},
$$


we have

$$
\begin{gathered}
(z-\tau)^{T} \cdot \boldsymbol{a} \int_{D} e^{x \cdot z-t(z \cdot z)} d x d t=\int_{D} \nabla_{(x, t)} \cdot\left(e^{x \cdot z-t(z \cdot z)} \boldsymbol{a}\right) d x d t \\
=\sum_{j=1}^{3} \boldsymbol{a} \cdot\left(\boldsymbol{\nu}_{j} 0\right)^{T} \int_{\Delta_{j}} e^{x \cdot z-t(z \cdot z)} d S(x, t)+\boldsymbol{a} \cdot(0-1)^{T} \int_{\Delta_{4}} e^{x \cdot z-t(z \cdot z)} d S(x, t) \\
-\boldsymbol{a} \cdot \omega(c) \int_{Q} e^{x \cdot z-t(z \cdot z)} d S(x, t)
\end{gathered}
$$

where we made use of the fact that the unit outward normal vector to $\partial D$ takes $\left(\boldsymbol{\nu}_{j} 0\right)^{T}$ on $\Delta_{j}$ for each $j=1,2,3 ;-\boldsymbol{e}_{4}=(0-1)^{T}$ on $\Delta_{4} ;-\omega(c)$ on $Q$. Since $\boldsymbol{a}$ is arbitrary, one obtains

$$
\begin{gathered}
\left(\begin{array}{c}
z \\
-\tau
\end{array}\right) \int_{D} e^{x \cdot z-t(z \cdot z)} d x d t \\
=\sum_{j=1}^{3}\left(\begin{array}{c}
\boldsymbol{\nu}_{j} \\
0
\end{array}\right) \int_{\Delta_{j}} e^{x \cdot z-t(z \cdot z)} d S(x, t)+\left(\begin{array}{c}
0 \\
-1
\end{array}\right) \int_{\Delta_{4}} e^{x \cdot z-t(z \cdot z)} d S(x, t) \\
-\omega(c) \int_{Q} e^{x \cdot z-t(z \cdot z)} d S(x, t) .
\end{gathered}
$$

Since $Q$ is included in the hyper plane $(x t)^{T} \cdot \omega(c)=\left(x_{0} t_{0}\right)^{T} \cdot \omega(c)-\delta$, we have

$$
e^{-x_{0} \cdot z+t_{0}(z \cdot z)} \omega(c) \int_{Q} e^{x \cdot z-t(z \cdot z)} d S(x, t)=O\left(e^{-\tau \delta \sqrt{1+c^{2}}}\right) .
$$

We compute the integral

$$
I_{j}(\tau)=\int_{\Delta_{j}} e^{x \cdot z-t(z \cdot z)} d S(x, t), j=1,2,3,4 .
$$

On $\Delta_{j}$ for each $j$ one can write

$$
\left(\begin{array}{c}
x \\
t
\end{array}\right)=\left(\begin{array}{c}
x_{0} \\
t_{0}
\end{array}\right)+\alpha \boldsymbol{a}_{j}+\beta \boldsymbol{b}_{j}+\gamma \boldsymbol{c}_{j},
$$

where $(\alpha, \beta, \gamma) \in \Delta_{0}=\{(\alpha, \beta, \gamma) \mid \alpha+\beta+\gamma \leq 1, \alpha, \beta, \gamma \geq 0\}$ and $\boldsymbol{a}_{j}, \boldsymbol{b}_{j}, \boldsymbol{c}_{j}$ are suitable linearly independent vectors in $\mathbf{R}^{3+1}$ and satisfy the condition

$$
\boldsymbol{a}_{j} \cdot \omega(c)<0, \boldsymbol{b}_{j} \cdot \omega(c)<0, \boldsymbol{c}_{j} \cdot \omega(c)<0 .
$$

Writing $A_{j}=\left(\begin{array}{lll}\boldsymbol{a}_{j} & \boldsymbol{b}_{j} & \boldsymbol{c}_{j}\end{array}\right)$ which is a $4 \times 3$-matrix and $\tau \Delta_{0}=\{(\alpha, \beta, \gamma) \mid \alpha+\beta+\gamma \leq$ $\tau, \alpha, \beta, \gamma \geq 0\}$, we have

$$
\begin{gathered}
e^{-x_{0} \cdot z+t_{0}(z \cdot z)} I_{j}(\tau)=\sqrt{\operatorname{det} A_{j}^{T} A_{j}} \int_{\Delta_{0}} e^{\left(\alpha \boldsymbol{a}_{j}+\beta \boldsymbol{b}_{j}+\gamma \boldsymbol{c}_{j}\right) \cdot(z-\tau)^{T}} d \alpha d \beta d \gamma \\
=\frac{1}{\tau^{3}} \int_{\tau \Delta_{0}} e^{\left(\alpha \boldsymbol{a}_{j}+\beta \boldsymbol{b}_{j}+\gamma \boldsymbol{c}_{j}\right) \cdot\left(c\left(\omega+i \sqrt{1-\left(1 / c^{2} \tau\right)} \omega^{\perp}\right)-1\right)^{T}} d \alpha d \beta d \gamma
\end{gathered}
$$


Thus together with (4.18) yields

$$
\begin{gathered}
\lim _{\tau \longrightarrow \infty} \tau^{3} e^{-x_{0} \cdot z+t_{0}(z \cdot z)} I_{j}(\tau)=\int_{0}^{\infty} d \alpha \int_{0}^{\infty} d \beta \int_{0}^{\infty} d \gamma e^{\left(\alpha \boldsymbol{a}_{j}+\beta \boldsymbol{b}_{j}+\gamma \boldsymbol{c}_{j}\right) \cdot \boldsymbol{\vartheta}} \\
=\frac{(-1)^{3}}{\left(\boldsymbol{a}_{j} \cdot \boldsymbol{\vartheta}\right)\left(\boldsymbol{b}_{j} \cdot \boldsymbol{\vartheta}\right)\left(\boldsymbol{c}_{j} \cdot \boldsymbol{\vartheta}\right)}
\end{gathered}
$$

where $\boldsymbol{\vartheta}=\left(c\left(\omega+i \omega^{\perp}\right)-1\right)^{T}$.

From (4.16), (4.17), (4.19) we obtain

$$
\begin{gathered}
\lim _{\tau \longrightarrow \infty}\left(\begin{array}{c}
z \\
-\tau
\end{array}\right) \tau^{3} e^{-x_{0} \cdot z+t_{0}(z \cdot z)} \int_{D} e^{x \cdot z-t(z \cdot z)} d x d t \\
=-\sum_{j=1}^{3} \frac{\sqrt{\operatorname{det} A_{j}^{T} A_{j}}}{\left(\boldsymbol{a}_{j} \cdot \boldsymbol{\vartheta}\right)\left(\boldsymbol{b}_{j} \cdot \boldsymbol{\vartheta}\right)\left(\boldsymbol{c}_{j} \cdot \boldsymbol{\vartheta}\right)}\left(\begin{array}{c}
\boldsymbol{\nu}_{j} \\
0
\end{array}\right)-\frac{\sqrt{\operatorname{det} A_{4}^{T} A_{4}}}{\left(\boldsymbol{a}_{4} \cdot \boldsymbol{\vartheta}\right)\left(\boldsymbol{b}_{4} \cdot \boldsymbol{\vartheta}\right)\left(\boldsymbol{c}_{4} \cdot \boldsymbol{\vartheta}\right)}\left(\begin{array}{c}
0 \\
-1
\end{array}\right) .
\end{gathered}
$$

This yields the formula

$$
\lim _{\tau \longrightarrow \infty} e^{-x_{0} \cdot z+t_{0}(z \cdot z)} \tau^{4} \int_{D} e^{x \cdot z-t(z \cdot z)} d x d t=-\frac{\sqrt{\operatorname{det} A_{4}^{T} A_{4}}}{\left(\boldsymbol{a}_{4} \cdot \boldsymbol{\vartheta}\right)\left(\boldsymbol{b}_{4} \cdot \boldsymbol{\vartheta}\right)\left(\boldsymbol{c}_{4} \cdot \boldsymbol{\vartheta}\right)}
$$

By choosing $\rho \equiv 1$ in Proposition 4.2, one concludes

$$
K_{D}=-\frac{2}{3 !} c^{4} \frac{\sqrt{\operatorname{det} A_{4}^{T} A_{4}}}{\left(\boldsymbol{a}_{4} \cdot \boldsymbol{\vartheta}\right)\left(\boldsymbol{b}_{4} \cdot \boldsymbol{\vartheta}\right)\left(\boldsymbol{c}_{4} \cdot \boldsymbol{\vartheta}\right)} .
$$

Therefore $D$ is visible at $\left(x_{0}, t_{0}\right)$ from the complex direction $z$ and $(4.7)$ is valid for $\mu=4$ and $C$ given by the formula

$$
C=-\frac{\sqrt{\operatorname{det} A_{4}^{T} A_{4}}}{\left(\boldsymbol{a}_{4} \cdot \boldsymbol{\vartheta}\right)\left(\boldsymbol{b}_{4} \cdot \boldsymbol{\vartheta}\right)\left(\boldsymbol{c}_{4} \cdot \boldsymbol{\vartheta}\right)}
$$

Therefore (4.11) is valid for this $C$ and $\mu=4$ provided $\delta$ is chosen in such a way that $\left.\overline{D\left(x_{0}, x_{1}, x_{2}, x_{3}, \omega(c), \delta\right)} \subset \Omega \times\right] 0, T[$.

\section{$5 \quad$ An integral representation of $G_{z}$ and a byproduct}

It is quite important for us to compute $v$ given by (3.18) with $g=\chi_{D}$. In this section we give an integral representation of the distribution $G_{z}(x, t)$ together with

$$
K_{z}(x, t)=e^{x \cdot z-t(z \cdot z)} G_{z}(x, t), z=\boldsymbol{a}+i \boldsymbol{b}, \boldsymbol{b} \neq 0
$$

which is a solution of the equation $\partial_{t} v+\Delta v+\delta(x, t)=0$ in $\mathbf{R}^{n+1}$. Using the representation of $K_{z}$, we show that $K_{z}$ in the hyper space $(x t)^{T} \cdot \omega(c)<0$ with $z$ given by (4.6) is exponentially decaying as $\tau \longrightarrow \infty$. As a byproduct of this fact we see that $K_{z}$ yields a Carleman type formula for the heat equation. 


\subsection{Representation of $G_{z}$}

Proposition 5.1. It holds that

$$
\begin{gathered}
G_{z}(x, t) \\
=e^{-i(x-2 t \boldsymbol{a}) \cdot \boldsymbol{b}-|\boldsymbol{b}|^{2} t} \times \\
\left\{-\left(\frac{|\boldsymbol{b}|}{2 \pi}\right)^{n} \int_{|\xi|<1} e^{i|\boldsymbol{b}|(x-2 t \boldsymbol{a}) \cdot \xi_{e}|\xi|^{2}|\boldsymbol{b}|^{2} t} d \xi+H(-t)\left(\frac{1}{2 \sqrt{\pi|t|}}\right)^{n} e^{\frac{|x-2 t \boldsymbol{a}|^{2}}{4 t}}\right\} .
\end{gathered}
$$

Proof. First we give a representation for $F_{1}(x, t)=G_{i \omega}(x, t)$ where $\omega=\boldsymbol{b} /|\boldsymbol{b}|$. The starting point is the following formulae for the Fourier transform:

Let $H(t)$ denote the Heaviside function. Let $\operatorname{Re} c \neq 0$. It is easy to see that: if $\operatorname{Re} c>0$, then

$$
\frac{1}{-i \eta-c}=-\int H(t) e^{-c t} e^{-i t \eta} d t
$$

if $\operatorname{Re} c<0$, then

$$
\frac{1}{-i \eta-c}=\int H(-t) e^{-c t} e^{-i t \eta} d t .
$$

Thus $F_{1}(x, t)$ becomes

$$
\begin{gathered}
F_{1}(x, t)=\frac{1}{(2 \pi)^{(n+1)}} \int e^{i(x \cdot \xi)+i t \eta} \frac{d \xi d \eta}{-i \eta-\left(1-|\xi+\omega|^{2}\right)} \\
=\frac{1}{(2 \pi)^{n}}\left\{\int_{|\xi+\omega|<1} e^{i x \cdot \xi}\left\{-H(t) e^{\left(|\xi+\omega|^{2}-1\right) t}\right\} d \xi+\int_{|\xi+\omega|>1} e^{i x \cdot \xi}\left\{H(-t) e^{\left(|\xi+\omega|^{2}-1\right) t}\right\} d \xi\right\} \\
=-\frac{H(t)}{(2 \pi)^{n}} \int_{|\xi+\omega|<1} e^{i x \cdot \xi} e^{\left(|\xi+\omega|^{2}-1\right) t} d \xi+\frac{H(-t)}{(2 \pi)^{n}} \int_{|\xi+\omega|>1} e^{i x \cdot \xi} e^{\left(|\xi+\omega|^{2}-1\right) t} d \xi .
\end{gathered}
$$

This together with a change of variables yields

$$
\begin{gathered}
F_{1}(x, t) \\
=-\frac{H(t) e^{-i x \cdot \omega}}{(2 \pi)^{n}} \int_{|\xi|<1} e^{i x \cdot \xi} e^{\left(|\xi|^{2}-1\right) t} d \xi+\frac{H(-t) e^{-i x \cdot \omega}}{(2 \pi)^{n}} \int_{|\xi|>1} e^{i x \cdot \xi} e^{\left(|\xi|^{2}-1\right) t} d \xi
\end{gathered}
$$

and thus we obtain

$$
\begin{gathered}
e^{i x \cdot \omega+t} F_{1}(x, t) \\
=-\frac{H(t)}{(2 \pi)^{n}} \int_{|\xi|<1} e^{i x \cdot \xi} e^{|\xi|^{2} t} d \xi+\frac{H(-t)}{(2 \pi)^{n}} \int_{|\xi|>1} e^{i x \cdot \xi} e^{|\xi|^{2} t} d \xi .
\end{gathered}
$$

Since

$$
\int e^{i x \cdot \xi} e^{-|\xi|^{2} a} d \xi=\left(\sqrt{\frac{\pi}{a}}\right)^{n} e^{-\frac{|x|^{2}}{4 a}}, a>0,
$$


we have, for all $t<0$

$$
\begin{gathered}
\frac{1}{(2 \pi)^{n}} \int_{|\xi|>1} e^{i x \cdot \xi} e^{|\xi|^{2} t} d \xi=\frac{1}{(2 \pi)^{n}}\left\{\left(\sqrt{\frac{\pi}{|t|}}\right)^{n} e^{\frac{|x|^{2}}{4 t}}-\int_{|\xi|<1} e^{i x \cdot \xi} e^{|\xi|^{2} t} d \xi\right\} \\
=\left(\frac{1}{2 \sqrt{\pi|t|}}\right)^{n} e^{\frac{|x|^{2}}{4 t}}-\frac{1}{(2 \pi)^{n}} \int_{|\xi|<1} e^{i x \cdot \xi} e^{|\xi|^{2} t} d \xi .
\end{gathered}
$$

This together with (5.3) yields

$$
\begin{gathered}
e^{i x \cdot \omega+t} F_{1}(x, t) \\
=-\frac{1}{(2 \pi)^{n}}\{H(t)+H(-t)\} \int_{|\xi|<1} e^{i x \cdot \xi} e^{|\xi|^{2} t} d \xi+H(-t)\left(\frac{1}{2 \sqrt{\pi|t|}}\right)^{n} e^{\frac{|x|^{2}}{4 t}} \\
=-\frac{1}{(2 \pi)^{n}} \int_{|\xi|<1} e^{i x \cdot \xi} e^{|\xi|^{2} t} d \xi+H(-t)\left(\frac{1}{2 \sqrt{\pi|t|}}\right)^{n} e^{\frac{|x|^{2}}{4 t}} .
\end{gathered}
$$

It follows from (3.3) that

$$
G_{i \boldsymbol{b}}(x, t)=|\boldsymbol{b}|^{n} F_{1}\left(|\boldsymbol{b}| x,|\boldsymbol{b}|^{2} t\right) .
$$

A combination of this and (5.5) gives

$$
\begin{gathered}
G_{i \boldsymbol{b}}(x, t) \\
=e^{-i x \cdot \boldsymbol{b}-|\boldsymbol{b}|^{2} t}\left\{-\left(\frac{|\boldsymbol{b}|}{2 \pi}\right)^{n} \int_{|\xi|<1} e^{i|\boldsymbol{b}| x \cdot \xi_{e}|\xi|^{2}|\boldsymbol{b}|^{2} t} d \xi+H(-t)\left(\frac{1}{2 \sqrt{\pi|t|}}\right)^{n} e^{\frac{|x|^{2}}{4 t}}\right\} .
\end{gathered}
$$

From this we immediately obtain (5.2) since we have (3.13).

\subsection{Representation of $K_{z}$}

From (5.1), (5.2) and the equation

$$
x \cdot \boldsymbol{a}-t|\boldsymbol{a}|^{2}+\frac{1}{4 t}|x-2 t \boldsymbol{a}|^{2}=\frac{|x|^{2}}{4 t},
$$

it follows that

$$
K_{z}(x, t)=H(-t)\left(\frac{1}{2 \sqrt{\pi|t|}}\right)^{n} e^{\frac{|x|^{2}}{4 t}}+w_{z}(x, t)
$$

where

$$
w_{z}(x, t)=-e^{x \cdot \boldsymbol{a}-t|\boldsymbol{a}|^{2}}\left(\frac{|\boldsymbol{b}|}{2 \pi}\right)^{n} \int_{|\xi|<1} e^{i|\boldsymbol{b}|(x-2 t \boldsymbol{a}) \cdot \xi} e^{|\boldsymbol{b}|^{2}|\xi|^{2} t} d \xi
$$


Remarks are in order.

(i) Since the distribution

$$
H(-t)\left(\frac{1}{2 \sqrt{\pi|t|}}\right)^{n} e^{\frac{|x|^{2}}{4 t}}
$$

is the fundamental solution of the equation $\partial_{t} v+\Delta v=0$, the $w_{z}(x, t)$ is an entire solution of the backward heat equation. Moreover $w_{z}$ is a smooth function on the whole space.

(ii) From (5.8) and a change of variables we know that $w_{z}(x, t)=\overline{w_{z}(x, t)}$. Thus $w_{z}$ is real valued and hence we have

$$
w_{z}(x, t)=-e^{x \cdot \boldsymbol{a}-t|\boldsymbol{a}|^{2}}\left(\frac{|\boldsymbol{b}|}{2 \pi}\right)^{n} \int_{|\xi|<1} \cos (|\boldsymbol{b}|(x-2 t \boldsymbol{a}) \cdot \xi) e^{|\boldsymbol{b}|^{2}|\xi|^{2} t} d \xi .
$$

Note also that $w_{z}$ does not depend on the direction of the vector $\boldsymbol{b}$.

(iii) Write

$$
|\boldsymbol{b}|^{2}|\xi|^{2} t+i|\boldsymbol{b}|(x-2 t \boldsymbol{a}) \cdot \xi=t\left(|\boldsymbol{b}| \xi+i \frac{1}{2 t}(x-2 t \boldsymbol{a})\right)^{2}+\frac{1}{4 t}|x-2 t \boldsymbol{a}|^{2} .
$$

Combining this with (5.6), we can rewrite (5.8) as

$$
w_{z}(x, t)=-\frac{e^{|x|^{2} /(4 t)}}{(2 \pi)^{n}} \int_{|\xi|<|\boldsymbol{b}|} e^{t(\xi+i(x-2 t \boldsymbol{a}) /(2 t))^{2}} d \xi
$$

We study more the expression (5.7).

(i) The case when $t>0$. Since

$$
x \cdot \boldsymbol{a}-t|\boldsymbol{a}|^{2}+t|\boldsymbol{b}|^{2}=\tau \sqrt{1+c^{2}}\left(\begin{array}{c}
x \\
t
\end{array}\right) \cdot \omega(c),
$$

it follows from (5.7) and (5.8) that

$$
K_{z}(x, t)=-e^{\tau \sqrt{1+c^{2}}(x t)^{T} \cdot \omega(c)}\left(\frac{|\boldsymbol{b}|}{2 \pi}\right)^{n} \int_{|\xi|<1} e^{i|\boldsymbol{b}|(x-2 t \boldsymbol{a}) \cdot \xi} e^{-\left(1-|\xi|^{2}\right)|\boldsymbol{b}|^{2} t} d \xi
$$

(ii) The case when $t<0$. It follows from (5.4) that

$$
\begin{gathered}
\int_{|\xi|<1} e^{i|\boldsymbol{b}|(x-2 t \boldsymbol{a}) \cdot \xi_{e}|\boldsymbol{b}|^{2}|\xi|^{2} t} d \xi \\
=\frac{1}{|\boldsymbol{b}|^{n}}\left(\sqrt{\frac{\pi}{-t}}\right)^{n} e^{|x-2 t \boldsymbol{a}|^{2} /(4 t)}-\int_{|\xi|>1} e^{i|\boldsymbol{b}|(x-2 t \boldsymbol{a}) \cdot \xi_{e}|\boldsymbol{b}|^{2}|\xi|^{2} t} d \xi .
\end{gathered}
$$

This together with (5.7) and (5.8) yields

$$
K_{z}(x, t)=e^{\tau \sqrt{1+c^{2}}(x t)^{T} \cdot \omega(c)}\left(\frac{|\boldsymbol{b}|}{2 \pi}\right)^{n} \int_{|\xi|>1} e^{i|\boldsymbol{b}|(x-2 t \boldsymbol{a}) \cdot \xi_{e}|\boldsymbol{b}|^{2}\left(|\xi|^{2}-1\right) t} d \xi .
$$

Remark 5.1. Using the well known formula

$$
\int_{|\xi|=r} e^{i \eta \cdot \xi} d S(\xi)=(2 \pi)^{n / 2} r^{n / 2}|\eta|^{-(n-2) / 2} J_{(n-2) / 2}(|\eta| r), \forall \eta \in \mathbf{R}^{n},
$$

one can rewrite (5.8), (5.10) and (5.11) as one-dimensional integrals. 


\subsection{Exponential decaying of $K_{z}$ in the hyper space $(x t)^{T} \cdot \omega(c)<0$ and a Carleman type formula for the heat equation}

In this subsection first we show that $K_{z}(x, t)$ is exponentially decaying as $\tau \longrightarrow \infty$ if $(x t)^{T} \cdot \omega(c)<0$ and $z$ is given by $(4.6)$.

Proposition 5.2. Given $\delta>0$ we have, as $\tau \longrightarrow \infty$

$$
\sup _{(x t)^{T} \cdot \omega(c)<-\delta}\left|K_{z}(x, t)\right|=O\left(e^{-\tau \sqrt{1+c^{2}} \delta} \tau^{n}\right) .
$$

Proof. Let $(x, t)$ satisfy $(x t)^{T} \cdot \omega(c)<-\delta$.

(i) The case when $t>0$. From (5.10) we have immediately

$$
\left|K_{z}(x, t)\right| \leq C_{n} e^{-\tau \sqrt{1+c^{2}} \delta}|\boldsymbol{b}|^{n}
$$

(ii) The case when $t<0$. We divide this case into two subcases: (a) $|\boldsymbol{b}|^{2}(-t)$ is large; (b) $|\boldsymbol{b}|^{2}(-t)$ is not large and can be arbitrary small.

First consider (a). Given $R>0$ let $t$ satisfy $|\boldsymbol{b}|^{2} t<-R$. From (5.4) and (5.11) we obtain

$$
\left|K_{z}(x, t)\right| \leq e^{-\tau \sqrt{1+c^{2}} \delta}\left(\frac{|\boldsymbol{b}|}{2 \pi}\right)^{n} \int_{|\xi|>1} e^{-R\left(|\xi|^{2}-1\right)} d \xi \leq C_{n} e^{-\tau \sqrt{1+c^{2}}} \delta|\boldsymbol{b}|^{n} \frac{e^{R}}{R^{n / 2}} .
$$

Next consider (b). We employ the expression (5.7) and (5.8). Let $t$ satisfy $-R \leq$ $|\boldsymbol{b}|^{2} t<0$. Using (5.9), we can rewrite (5.8) as

$$
w_{z}(x, t)=-e^{\tau \sqrt{1+c^{2}}(x t)^{T} \cdot \omega(c)} e^{-|\boldsymbol{b}|^{2} t}\left(\frac{|\boldsymbol{b}|}{2 \pi}\right)^{n} \int_{|\xi|<1} e^{i|\boldsymbol{b}|(x-2 t \boldsymbol{a}) \cdot \xi} e^{|\boldsymbol{b}|^{2}|\xi|^{2} t} d \xi
$$

Since $t<0$ and $-|\boldsymbol{b}|^{2} t \leq R$, it follows from (5.14) that

$$
\left|w_{z}(x, t)\right| \leq C_{n} e^{-\tau \sqrt{1+c^{2}} \delta}|\boldsymbol{b}|^{n} e^{R} .
$$

Since $c x \cdot \omega<c x \cdot \omega-t<-\delta \sqrt{1+c^{2}}$, we have $|x \cdot \omega|>(\delta / c) \sqrt{1+c^{2}}$ and thus $|x|>(\delta / c) \sqrt{1+c^{2}}$. Using this together with $|\boldsymbol{b}|^{2}|t| \leq R$, we obtain

$$
\begin{gathered}
|t|^{-n} e^{|x|^{2} /(4 t)}=\left(|\boldsymbol{b}|^{2}|t|\right)^{-n}|\boldsymbol{b}|^{2 n} e^{-\left(|\boldsymbol{b}|^{2}|x|^{2}\right) /\left(4|\boldsymbol{b}|^{2}|t|\right)} \\
\leq \frac{|\boldsymbol{b}|^{2 n}}{R^{n}} e^{-\left(|\boldsymbol{b}|^{2}(\delta / c)^{2}\left(1+c^{2}\right)\right) /(4 R)}
\end{gathered}
$$

This together with (5.7) and (5.15) yields that

$$
\left|K_{z}(x, t)\right| \leq C_{n}\left(e^{-\tau \sqrt{1+c^{2}} \delta}|\boldsymbol{b}|^{n} e^{R}+\frac{|\boldsymbol{b}|^{2 n}}{R^{n}} e^{-\left(|\boldsymbol{b}|^{2}(\delta / c)^{2}\left(1+c^{2}\right)\right) /(4 R)}\right) .
$$

A combination of (5.13) and (5.16) gives

$$
\left|K_{z}(x, t)\right| \leq C_{n}\left\{e^{-\tau \sqrt{1+c^{2}}}|\boldsymbol{b}|^{n} e^{R}\left(\frac{1}{R^{n / 2}}+1\right)++\frac{|\boldsymbol{b}|^{2 n}}{R^{n}} e^{-\left(|\boldsymbol{b}|^{2}(\delta / c)^{2}\left(1+c^{2}\right)\right) /(4 R)}\right\} .
$$


Now Proposition 5.2 is a direct consequence of (5.12) and (5.17).

Remark 5.2. All the derivatives of $K_{z}(x, t)$ also have a similar property: for each $\alpha \in Z_{+}^{n}$ and $\beta \in \boldsymbol{Z}_{+}$

$$
e^{\tau \sqrt{1+c^{2}} \delta} \sup _{(x t)^{T} \cdot \omega(c)<-\delta}\left|\partial_{x}^{\alpha} \partial_{t}^{\beta} K_{z}(x, t)\right|
$$

is at most algebraically growing as $\tau \longrightarrow \infty$.

As a corollary of Proposition 5.1 and remark 5.2 we obtain a Carleman type formula.

Corollary 5.1. Let $\left.\left(x_{0}, t_{0}\right) \in \Omega \times\right] 0, T[$ be an arbitrary fixed point. Assume that $T>0$, $\omega, \Gamma$ and $U$ satisfy (4.8), (4.9) and (4.10). Let $v(x, t)=K_{z}\left(x-x_{0}, t-t_{0}\right)$ for $z$ given by (4.6) and $u$ be a solution of (2.7). Then we have

$$
u\left(x_{0}, t_{0}\right)=-\lim _{\tau \longrightarrow \infty} I(\tau)
$$

where

$$
I(\tau)=\int_{\Gamma}\left\{\left(\frac{\partial v}{\partial \nu}(x, t)+\rho(x) v(x, t)\right) u(x, t)-h_{0}(x, t) v(x, t)\right\} d S d t-\int_{U} v(x, 0) u(x, 0) d x .
$$

Remark 5.3. Yarmukhamedov [15] considered the Cauchy problem for the Laplace equation in a three-dimensional bounded domain $D$ that is bounded by the plane $x_{3}=0$ and by smooth surfaces lying in the half-space $x_{3}>0$. He gave a formula for calculating the value of the solution at a given point inside the domain from the Cauchy data on the portion in $x_{3}>0$ of $\partial D$. For the purpose he made use of a special fundamental solution for the Laplace operator which has been introduced by himself in [14] and is parameterized by an entire function $E(w), w \in C$ satisfying a suitable growth condition when $|\operatorname{Im} w| \longrightarrow \infty$. His fundamental solution $\Phi_{E}$ takes the form for $x=\left(x_{1}, x_{2}, x_{3}\right)$ with $x^{\prime}=\left(x_{1}, x_{2}\right) \neq 0$ :

$$
\Phi_{E}(x)=-\frac{1}{2 \pi^{2}} \int_{0}^{\infty} \operatorname{Im}\left(\frac{E\left(x_{3}+i \sqrt{\left|x^{\prime}\right|^{2}+u^{2}}\right)}{x_{3}+i \sqrt{\left|x^{\prime}\right|^{2}+u^{2}}}\right) \frac{d u}{\sqrt{\left|x^{\prime}\right|^{2}+u^{2}}} .
$$

He chose the special $E(w)=e^{\tau w}$ with $\tau>0$. Then $\Phi_{E}(x)$ has the representation

$$
\Phi_{E}(x)=-\frac{e^{\tau x_{3}}}{2 \pi^{2}} \int_{0}^{\infty}\left(x_{3} \frac{\sin \left(\tau \sqrt{\left|x^{\prime}\right|^{2}+u^{2}}\right)}{\sqrt{\left|x^{\prime}\right|^{2}+u^{2}}}-\cos \left(\tau \sqrt{\left|x^{\prime}\right|^{2}+u^{2}}\right)\right) \frac{d u}{|x|^{2}+u^{2}} .
$$

From this one sees that the Cauchy data of $\Phi_{E}(y-x)$ on the portion in $y_{3}=0$ of $\partial D$ for an arbitrary fixed $x \in D$ decays exponentially as $\tau \longrightarrow \infty$. This fact corresponds to Proposition 5.2 and is an evidence that the formula (5.18) can be considered as an extension to the heat equation of his formula.

It would be interesting to find a hidden 'parameter' for $K_{z}$ like $E$ for $\Phi_{E}$. That is: can one find a family of special fundamental solutions for the backward heat operator $\partial_{t}+\triangle$ that contains $K_{z}$ as a special member? In other words, can one find another fundamental solution for the backward heat operator that is decaying in one side of a hyper surface not plane? 


\section{Acknowledgement}

This research was partially supported by Grant-in-Aid for Scientific Research (C)(No. 18540160) of Japan Society for the Promotion of Science.

\section{References}

[1] Beck, J. V., Blackwell, B. and Clair, S. R., Inverse Heat Conduction. Ill-Posed Problems, Wiley, New York, 1985.

[2] Carasso, A., Determining surface temperatures from interior observations, SIAM J. Appl. Math., 42(1982), 558-574.

[3] Dautray, R. and Lions, J-L., Mathematical analysis and numerical methods for sciences and technology, Evolution problems I, Vol. 5, Berlin, Springer-Verlag, 1992.

[4] Eldén, L., Numerical solution of the sideways heat equation by difference approximation in time, Inverse Problems, 11(1995), 913-923.

[5] Hsieh, C.-C., Scattering theory for the operator $\frac{\partial}{\partial t}-\frac{\partial^{2}}{\partial x^{2}}-\frac{\partial^{2}}{\partial y^{2}}$, Inverse Problems, 18(2002), 153-174.

[6] Hörmander, L., The analysis of linear partial differential operators I, Springer-Verlag, 1983.

[7] Ikehata, M., A special Green's function for the biharmonic operator and its application to an inverse boundary value problem, Computers Math. Applic., 22(1991), 53-66.

[8] Ikehata, M., The enclosure method and its applications, in Analytic Extension Formulas and their Applications (ed Saitoh, S., Hayashi, N. and Yamamoto, M.), International Society for Analysis, Applications and Computation, Vol. 9, 87-103, Dordrecht, Kluwer Academic Publishers, 2001.

[9] Ikehata, M., Exponentially growing solutions and the Cauchy problem, Applicable Analysis, 78(2001), 79-95.

[10] Ikehata, M., An inverse source problem for the heat equation and the enclosure method, Inverse Problems, 23(2007), 183-202.

[11] Levine, H., Continuous data dependence, regularization, and a three lines theorem for the heat equation with data in a space like direction, Ann. Math. Pure Appl., (IV), CXXXIV(1983), 267-286.

[12] Lions, J. L. and Magenes, E., Non-Homogeneous boundary value problems and applications II, Springer, New York, 1972. 
[13] Sylvester, J. and Uhlmann, G., A global uniqueness theorem for an inverse boundary value problem, Ann. Math., 125(1987), 153-169.

[14] Yarmukhamedov, Sh., Integral representation of harmonic functions in multidimensions, Dokl. Akad. Nauk SSSR, 204(1972), 799-802.

[15] Yarmukhamedov, Sh., On a Cauchy problem for Laplace's equation, Mathematicheskie Zametki, 18(1975), No.1, 57-61.

e-mail address

ikehata@math.sci.gunma-u.ac.jp 